\title{
The Computational Fluid Dynamics Rupture Challenge 2013- Phase I: Prediction of Rupture Status in Intracranial Aneurysms
}

\author{
G. Janiga, P. Berg, S. Sugiyama, K. Kono, and D.A. Steinman
}

\begin{abstract}
BACKGROUND AND PURPOSE: Rupture risk assessment for intracranial aneurysms remains challenging, and risk factors, including wall shear stress, are discussed controversially. The primary purpose of the presented challenge was to determine how consistently aneurysm rupture status and rupture site could be identified on the basis of computational fluid dynamics.
\end{abstract}

MATERIALS AND METHODS: Two geometrically similar MCA aneurysms were selected, 1 ruptured, 1 unruptured. Participating computational fluid dynamics groups were blinded as to which case was ruptured. Participants were provided with digitally segmented lumen geometries and, for this phase of the challenge, were free to choose their own flow rates, blood rheologies, and so forth. Participants were asked to report which case had ruptured and the likely site of rupture. In parallel, lumen geometries were provided to a group of neurosurgeons for their predictions of rupture status and site.

RESULTS: Of 26 participating computational fluid dynamics groups, 21 (81\%) correctly identified the ruptured case. Although the known rupture site was associated with low and oscillatory wall shear stress, most groups identified other sites, some of which also experienced low and oscillatory shear. Of the 43 participating neurosurgeons, 39 (91\%) identified the ruptured case. None correctly identified the rupture site.

CONCLUSIONS: Geometric or hemodynamic considerations favor identification of rupture status; however, retrospective identification of the rupture site remains a challenge for both engineers and clinicians. A more precise understanding of the hemodynamic factors involved in aneurysm wall pathology is likely required for computational fluid dynamics to add value to current clinical decision-making regarding rupture risk.

ABBREVIATIONS: CFD = computational fluid dynamics; OSI = oscillatory shear index; Re = Reynolds; RRT = relative residence time; TAWSS = temporal-averaged wall shear stress; WSS $=$ wall shear stress

n case of a weakened cerebral vasculature, dilations of the vessel walls may form. It has been estimated that such intracranial aneurysms are present in approximately $2 \%-5 \%$ of the Western

Received March 7, 2014; accepted after revision September 9.

From the Department of Fluid Dynamics and Technical Flows (G.J., P.B.), University of Magdeburg, Magdeburg, Germany; Department of Neurosurgery (S.S.), Tohoku University Graduate School of Medicine, Miyagi, Japan; Department of Neurosurgery (K.K.), Wakayama Rosai Hospital, Wakayama, Japan; and Department of Mechanical and Industrial Engineering (D.A.S.), University of Toronto, Toronto, Ontario, Canada.

This work was partly funded by the Federal Ministry of Education and Research in Germany within the Research Campus STIMULATE under grant number 03FO16102A.

P.B. was supported by the International Max Planck Research School Magdeburg. D.A.S. was supported by a MidCareer Investigator award from the Heart and Stroke Foundation of Ontario.

Please address correspondence to Gábor Janiga, PhD, Laboratory of Fluid Dynamics and Technical Flows, University of Magdeburg "Otto von Guericke," Germany, Universitätsplatz 2, D-39106 Magdeburg, Germany; e-mail: janiga@ovgu.de

- Indicates open access to non-subscribers at www.ajnr.org

Indicates article with supplemental on-line tables.

Indicates article with supplemental on-line photo.

http://dx.doi.org/10.3174/ajnr.A4157 population. ${ }^{1,2}$ Slightly $<1 \%$ of these aneurysms rupture annually, but with mostly severe consequences. ${ }^{3}$ The mortality rate is up to $40 \%$, and even if treatment were available within hours, irreversible disabilities may remain afterward. ${ }^{4,5}$

However, if aneurysms are detected incidentally, the attending physician needs to suggest to the patient whether a treatment is necessary or a repeated observation is the best choice. Especially in complex case scenarios, the risk that treatment complications occur could be even higher than the potential risk of an aneurysm rupture itself. Therefore, any reliable assistance that can support the physician's therapy planning can improve the outcome for the patient.

During the past years, there has been success in using computational fluid dynamics (CFD) to predict intracranial aneurysm rupture status on the basis of a nominal relationship between local hemodynamics (notably wall shear stress, [WSS]) and aneurysmal wall remodeling and degradation. ${ }^{6-9}$ However, the retrospective prediction of rupture status (and, by extension, the prospective prediction of rupture risk) in intracranial aneurysms is 
controversially discussed in the literature. Xiang et $\mathrm{al}^{10}$ found that rupture status correlated with significantly lower WSS and a higher oscillatory shear index (OSI). In contrast, Cebral et $\mathrm{al}^{11}$ found that high maximum WSS and concentrated impingement regions might be responsible for an aneurysm rupture. In both studies, $>100$ intracranial aneurysms were investigated, and the authors tried to find correlations between hemodynamic or morphologic parameters and the known rupture status. Both studies agreed that ruptured aneurysms present complex flow structures and concentrated inflow. Recently, Meng et $\mathrm{al}^{12}$ and Xiang et al ${ }^{13}$ published a 2-part review summarizing the various criteria for rupture status predictions and introducing a hypothesis that both high and low WSS might lead to aneurysm rupture.

In the present study, we wanted to produce a snapshot of whether engineering groups are able to predict the event of an aneurysm rupture. Two of the authors (G.J. and D.A.S.) decided to propose a new computational challenge addressing 2 main questions: First, can an aneurysm rupture be predicted by using CFD? Second, along the lines of the previous American Society of Mechanical Engineering aneurysm CFD challenge, ${ }^{14}$ how broad is the variability of the different solution strategies used for the computations?

In Phase I, the participants were asked to predict which of the 2 provided aneurysms was ruptured and where the rupture site was located. The study was completely blinded; none of the CFD participants had information about the cases, and the source of the data was not communicated. For the second phase, participants were provided with prescribed viscosity and patient-specific mean velocity boundary conditions and were asked to repeat the simulations to separate solution variations from boundary condition variations. In the current work, only Phase I of this challenge and the corresponding results are presented. The variability of the CFD solutions is discussed in a complementary article. ${ }^{15}$

\section{MATERIALS AND METHODS Case Details}

One of the major difficulties was to find unpublished appropriate datasets in which the rupture site is known. One of the authors (S.S.) was able to provide 2 cases: an unruptured aneurysm with MRA examination every 6 months during 5 years and a ruptured one with a documented site of rupture. They were chosen on the basis of similar geometric characteristics of the MCA segments. The 2 intracranial aneurysms that were part of this rupture challenge were both located at the left M1 segment. On-line Table 1 summarizes the characteristics of both cases, which were not provided to participating CFD groups.

Clinical Presentation of Case 1. A 77-year-old female patient underwent a medical checkup of the brain, and MR angiography revealed an unruptured aneurysm (approximately $10 \mathrm{~mm}$ ) at the bifurcation of the left middle cerebral artery. Thereafter, the patient was followed up by MR angiography at 6-month intervals. Gradual growth of the aneurysm was observed during a 5-year follow-up; its form changed from spheric to irregular shape. Coil embolization was performed, considering the risk of rupture.

Clinical Presentation of Case 2. The chief symptom of the case 2 patient was sudden headache. CT examination determined the diagnosis of an SAH (Hunt and Kosnik grade II/World Federation of Neurosurgical Societies grade I). During the neck clipping surgery, the aneurysm was completely exposed except for the ruptured site, which was covered with a thick fibrin cap. The fibrin cap was removed gradually with much caution, but accidentally, intraoperative rupture occurred. Fig $1 A$ shows an image acquired during the intervention, with the rupture bleed clearly visible. Fig $1 B$ shows the reconstructed aneurysm model in a lateral view to visualize the nominal rupture site.

\section{Vascular Reconstruction}

The image acquisition was performed by using $3 \mathrm{D}$ digital subtraction angiography. Digital segmentation was performed in Magdeburg, Germany, to fix the computational geometry for all participants. ${ }^{16}$ A neuroradiologist reviewed the virtual geometries afterward to check the plausibility of the reconstructed shapes. The obtained surface geometries were provided to the participants as surface triangulation mesh files. On-line Fig 1 shows the 2 investigated cases.

\section{Participating Groups}

The first phase of the CFD Rupture Challenge 2013 started on July 1 , and all groups that were willing to participate had the opportunity to submit the requested data until August 23. The complete announcement can be accessed on http://www.ovgu.de/isut/LSS/ CFD/CFD_Challenge.html. More than 30 groups from academia and industry accepted the challenge and asked for the reconstructed surface geometries. Ultimately, 26 submitted a short abstract describing the details of their computations and their rupture predictions. They were from 15 countries and 4 different continents; therefore, the challenge indeed represented a worldwide snapshot in computational hemodynamics of intracranial aneurysms. The participating groups are listed in On-line Table 2.

\section{CFD Methods}

Often in CFD challenges, very strict conditions are provided (see, eg, Steinman et $\mathrm{al}^{14}$ ) to test the variability of the solution in the absence of other sources of measurement variability. However, because a variety of rupture-status-predicting approaches exist throughout the literature, we decided in advance to provide the surface models of the intracranial aneurysms exclusively. Therefore, each group had the freedom to choose any desired volume mesh with arbitrary types of elements and resolution, flow/velocity boundary conditions, solver methodology, blood rheology, and so forth. With this flexibility, the participants were asked solely to report which of the 2 cases had ruptured and where the site of rupture was located.

Most the simulations (16/26) were performed by using commercial CFD solvers, but open-source and in-house codes were also applied. Ten groups decided to use steady-state computations; 14 groups used unsteady simulations for their predictions, while 2 groups performed both. The unsteady computations were performed by using various time-steps in the range of $\Delta t=1 \times$ $10^{-5}$ seconds to $5 \times 10^{-3}$ seconds (ie, 100,000 down to 200 timesteps per cardiac cycle) with a median of $7.5 \times 10^{-4}$ seconds $(\sim 1300$ steps per cardiac cycle). The reported mesh sizes were in the range of 283,000 to 17.97 million linear elements (median, 2.1 million). These figures included 3 groups that used quadratic tet- 

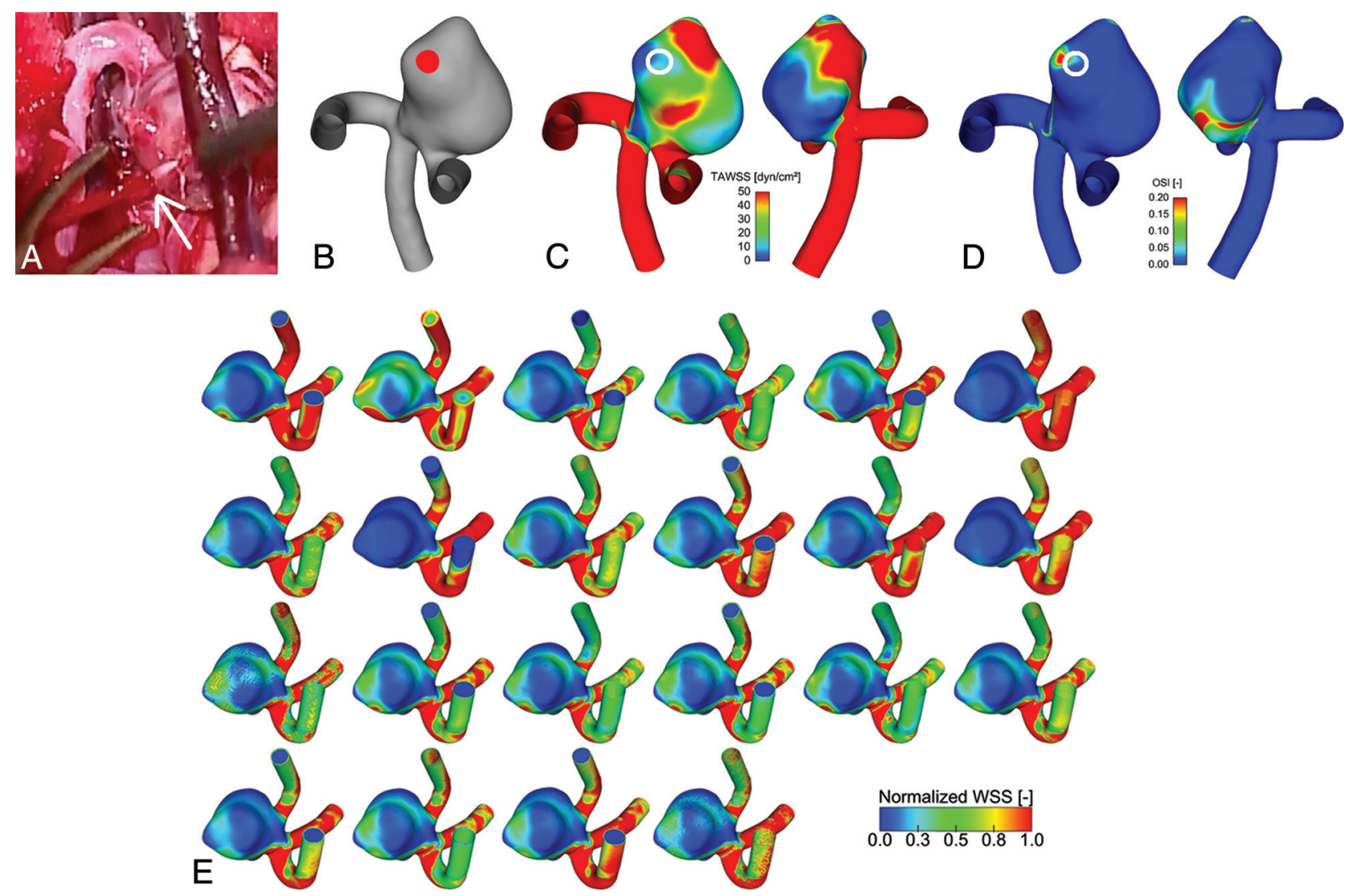

FIG 1. A, Live image acquired during open surgery showing the rupture that occurred in case 2 . The white arrow indicates the rupture site from which blood is streaming. $B$, Reconstructed surface model of case 2 indicating the ruptured site. $C$, Temporal averaged wall shear stress. $D$, Oscillatory shear index from 1 representative group, in 2 opposite perspectives. The site of rupture is indicated by a white circle. A low shear and elevated oscillating shear area are present. E, Sagittal view of the normalized wall shear stress distribution in the ruptured case 2 predicted by 22 groups with different simulation settings. Although most of the solutions qualitatively agree, considerable differences in the shear patterns are visible, depending on the type of simulation.

rahedral elements, in which case their number of elements was multiplied by 8 to indicate the effective number of linear elements. Mesh-dependency studies were reported by 5 groups.

As a preparation of the hemodynamic simulation, 7 groups extruded the inlet. Sixteen groups imposed a uniform (plug) velocity profile at the inlet of the computational domain, while 7 groups used Poiseuille (parabolic) velocity profiles and 3 groups used Womersley profiles. Nineteen groups reported their chosen flow rates, inlet velocities, or Reynolds (Re) numbers. Most applied cycle-average flow rates ranging from 0.9 to $11.36 \mathrm{~mL} / \mathrm{s}$ with a median of $3.5 \mathrm{~mL} / \mathrm{s}$. The corresponding inlet velocities were between 0.25 and $1.51 \mathrm{~m} / \mathrm{s}$ (median, $0.61 \mathrm{~m} / \mathrm{s}$ ) with the minimum $/$ maximum/ median Re numbers of 134/815/326. Unrealistic values were also reported (eg, Reynolds number, 1; inlet velocity, $10 \mathrm{~m} / \mathrm{s}$ ). The corresponding patient-specific flow rates were provided in the second phase of the CFD challenge. The cycle-averaged flow rates were 1.76 $\mathrm{mL} / \mathrm{s}$ for case 1 and $2.5 \mathrm{~mL} / \mathrm{s}$ for case 2 , whereas the peak systolic flow rates were 2.61 and $3.88 \mathrm{~mL} / \mathrm{s}$, respectively. See the Phase II study ${ }^{15}$ for further details regarding the flow measurements.

Regarding the outlet boundary condition, nearly one-half $(n=12)$ defined zero pressure at all outlets. Another 12 groups applied an explicit flow splitting through the 2 outlets (eg, 50-50, or based on Murray's law). Only 2 groups implemented a 3-element Windkessel model for the downstream microvasculature.
Most of the participants ( $n=18$ ) assumed blood to be an incompressible Newtonian fluid, with a dynamic viscosity ranging from 3.5 to $4.01 \mathrm{mPa} \cdot \mathrm{s}$ (median, $4 \mathrm{mPa} \cdot \mathrm{s}$ ). The other groups described the shear dependency of blood with a Carreau-Yasuda, Cross, or power law model. The survey of all abstracts revealed that a wide variety of approaches was chosen; therefore, the field of CFD was highly represented.

\section{Prediction of Rupture Status and Rupture Site}

Various criteria were selected for the rupture-status assessment and, in consequence, the prediction of the rupture site. The most common variable was temporal-averaged wall shear stress (TAWSS) or just WSS in case of steady computations, but in some predictions, uncommon criteria (eg, the maximum velocity at the ostium) were selected. A few groups applied a single criterion for their prediction, while most combined 2 or 3 criteria simultaneously. The parameters that were used to predict the explicit rupture site are summarized in the Table. Redundant variables were also selected (ie, high relative residence time [RRT] is a direct consequence of low WSS [and high OSI]). ${ }^{17}$

\section{Wall Shear Stress Computation}

In addition to the rupture prediction, all participants were asked to submit their solution of the 3D TAWSS distribution. There- 
fore, the variability of the WSS computation could be assessed directly. Another purpose was to ensure that the participating groups had actually performed the simulations that informed their prediction of rupture status and site. The submitted WSS distributions have shown very different maximum values, possibly due to the different near-wall resolutions but more likely because of the very different flow rates applied at the inlet. Because the results could not be compared dimensionally, each dataset was cropped at a chosen location of the parent artery and the calculated WSS field was normalized by the maximum value in the sac, to compare the relative qualitative distributions of WSS.

\section{Neurosurgeon Challenge}

One of the authors (K.K.), who performs endovascular treatments, open skull surgery, and CFD simulations on cerebral aneurysms, thought that it would be interesting to know the prediction of the ruptured aneurysm and the rupture site by neurosurgeons versus computer simulations. A single-page questionnaire (On-line Fig 3) was created providing only 3D images of the 2 aneurysms in 4 different angles. The questionnaire included the following: maximum size, neck size, height, diameters of the aneurysm in 3 axes, and size of the parent arteries. No other information, including the patient data, risk factors, and treatments

\begin{tabular}{|c|c|c|}
\hline $\begin{array}{c}\text { No. of } \\
\text { Parameters }\end{array}$ & Parameter & $\begin{array}{l}\text { No. of } \\
\text { Groups }\end{array}$ \\
\hline \multirow[t]{4}{*}{1} & Low (TA)WSS & 6 \\
\hline & High (TA)WSS & 3 \\
\hline & High pressure & 1 \\
\hline & Maximum velocity & 1 \\
\hline \multirow[t]{6}{*}{2} & Low (TA)WSS, high pressure & 3 \\
\hline & Low (TA)WSS, high OSI & 3 \\
\hline & High (TA)WSS, pressure gradient & 2 \\
\hline & Low TAWSS, complex flow & 1 \\
\hline & High TAWSS, complex flow & 1 \\
\hline & High TAWSS, direct impingement & 1 \\
\hline \multirow[t]{3}{*}{3} & Low TAWSS, high OSI, high RRT & 1 \\
\hline & TAWSS distribution, complex flow, flow diversion & 1 \\
\hline & Direct impingement, pressure gradient, high WSS & 1 \\
\hline
\end{tabular}

Note:-TAWSS indicates time-averaged WSS values; (TA)WSS, both steady-state as well as time-dependent results.

\section{Case 1} $n=4(15 \%)$

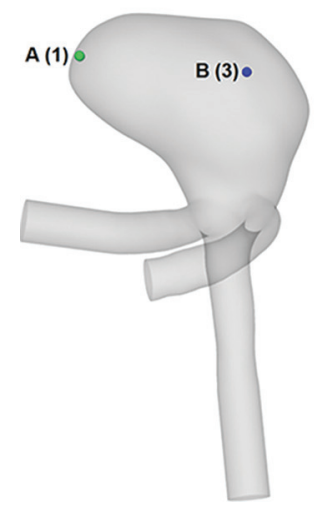

presented in On-line Table 1, was available. As noted above, both aneurysms had been carefully selected in advance to have comparable dimensions. K.K. asked 43 neurosurgeons in Japan to predict the ruptured aneurysm and the rupture site using the questionnaire. Additionally, they were asked to report the years of experience as a neurosurgeon and the reasons for the prediction of the ruptured aneurysm and the rupture point. The questionnaire had been completed before the CFD simulations were performed and the ruptured case identified. Thus, this questionnaire was performed in a double-blinded fashion.

\section{RESULTS}

\section{Prediction of Rupture Status and Rupture Site}

Regarding the challenge in Phase I, 4 groups predicted rupture for case 1, 21 groups chose case 2, and 1 group was unsure and therefore did not contribute any predictions. The ruptured aneurysm was case 2 as described above, so most, 21 of 26 groups (81\%), predicted the ruptured aneurysm correctly. As illustrated in Fig 2, the predictions of the site of rupture for case 1 concentrated on 2 locations. Three of the 4 groups decided that the impingement zone had the highest probability of being the rupture site $(B$, blue). High WSS and an elevated pressure gradient were associated with this area. Only 1 group suggested low WSS at the dome of the first aneurysm to be responsible for the rupture ( $A$, green). A broader variability was present for (the ruptured) case 2. Here, the majority $(n=10)$ derived from their computations that the rupture occurred at the small bleb, which was located at the dome of case 2 ( $C$, blue) and exposed to low WSS and elevated pressure. The second highest number of predictions picked the neck region that was aligned with the entering flow jet $(F$, red). For all 4 of those groups, the decision was based on high WSS, whereby 2 groups added the pressure gradient as a criterion for rupture. Another 4 groups associated the site of rupture with areas between the neck and dome of the aneurysm ( $E$, green, and $G$, purple). They made their choice on the basis of areas of low WSS and high OSI, whereby multiple spots with high oscillatory shear were identified (Fig 1D). Two more groups selected the impingement zone where the entering flow jet is directed to the aneurysm wall ( $D$, cyan). Finally, 1 group chose the end of the parent artery

\section{Case 2} $\mathrm{n}=21(81 \%)$

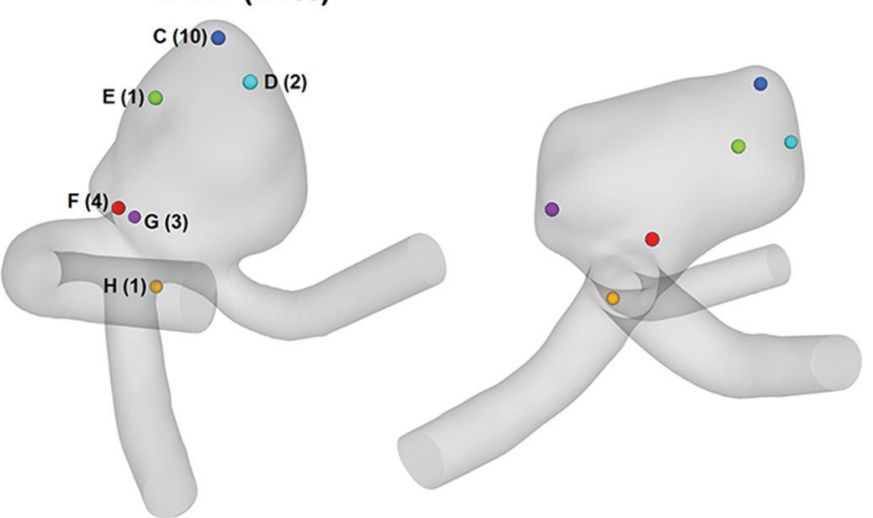

FIG 2. Sites of rupture predicted by the participating groups for case 1 and case 2 ( 3 perspectives). From the 26 submissions, 4 chose case 1 and 21 decided that case 2 was ruptured. One group was unsure and declined to provide a decision. 
because the highest velocities were present due to the smallest vessel diameter ( $H$, orange). However, after we reviewed the submitted abstract of this particular group, the results can be classified as unrealistic (peak velocity as rupture criterion; peak-systolic inflow velocity of $10 \mathrm{~m} / \mathrm{s}$ for both cases).

Throughout the complete challenge period, both the participants and the organizers were blinded regarding the rupture solution to ensure objective conditions. Afterward, author S.S. revealed the ruptured case.

The known site of rupture can now be compared with hemodynamic parameters that are typically used to characterize aneurysmal flow. We focused on TAWSS and OSI as major representatives of the predictions (Table). The results of wall shear stress and the oscillatory shear index were taken from a group that implemented inflow conditions that have been close to patient-specific. As indicated in Fig $1 C$, the ruptured site is located in an area that experiences rather low TAWSS compared with the parent artery with values well above $50 \mathrm{dyn} / \mathrm{cm}^{2}$. In the indicated region, TAWSS ranges between 0.1 and $19 \mathrm{dyn} / \mathrm{cm}^{2}$. Furthermore, elevated OSI is visible within the region of interest (Fig 1D). However, very low WSS $\left(<5 \mathrm{dyn} / \mathrm{cm}^{2}\right)$ and high oscillatory shear with values up to 0.42 are present beside this area.

\section{WSS Distributions}

WSS distributions contributed by the participants are shown in Fig $1 E$ (for the ruptured case 2). The WSS distributions for the unruptured case 1 are presented in On-line Fig 4. The WSS patterns on the aneurysm surface are obtained from 22 participants that used different assumptions to calculate the hemodynamic parameters. The normalized values demonstrate qualitative similarities, but differences regarding, for example, the size of different regions can also be observed. These differences show the variability of CFD and might explain the variability of rupture site prediction.

The different WSS predictions revealed the importance of realistic boundary conditions such as patient-specific measurements. This information was provided to all participants in a second phase along with further simulation conditions. The corresponding results, which represent the inherent variability of the CFD solutions among all groups, are not discussed in this article but are extensively described in the complementary work. $^{15}$

\section{Neurosurgeon Predictions}

The mean experience of neurosurgeons was $14.5 \pm 9.4$ years. Most of the neurosurgeons (39/43) correctly predicted that the case 2 aneurysm was the ruptured one because of blebs (56\%) or irregular shape (44\%). The predictions of the rupture site were split (On-line Fig 2). The main reasons for prediction of the rupture site in case 2 were impact zone (42\%) or tip of the aneurysm (58\%) for point B (red) and the irregular and sharp bleb (75\%) or the flow stagnation area (15\%) for point C (blue), respectively. None predicted the rupture site correctly.

\section{DISCUSSION}

The results of the challenge were first presented in a plenary talk at the IntraCranial Stent Meeting, which was held as a joint meeting with the World Federation of Interventional and Therapeutic Neuroradiology Congress in November 2013 in Buenos Aires. At the beginning of the talk, only the attending neurosurgeons and neuroradiologists from the whole audience were polled to predict the rupture status before seeing the results of the challenge. Five selected case 1 , while $>20$ chose case 2 . The clinicians from the audience were shown only the geometry of both cases and On-line Table 1 (without the geometric information). That table was not available for the participants during the challenge (engineers and neurosurgeons). Case 1 is slightly larger, while for case 2, smoking and hypertension should be mentioned. This information might have influenced the ad hoc on-site poll. The ratio of the predictions was very similar regarding both the engineering participants from the CFD challenge and the clinicians at the conference and from the independent neurosurgeon questionnaire.

One of the goals of CFD research is to make CFD simulations clinically useful. However, CFD simulations still seem far from clinical use. There are only a few reports in which CFD simulations are used for designing treatment strategy. ${ }^{18} \mathrm{CFD}$ simulations would not become helpful if they predict a rupture in the same aneurysms as clinicians already do. CFD simulations can be useful only when they change neurosurgeons' decisions or treatment strategy or add helpful hemodynamic information that is inaccessible to physicians. In the current CFD and neurosurgeon challenge, which consists of 2 cases only, prediction by computers and neurosurgeons was comparable. Prediction by CFD simulations took hours, while prediction by neurosurgeons took $<1$ minute. Thus, in this particular situation at least, neurosurgeons would not use CFD in the clinical field. One method to clarify possible clinical applications of computer simulations is to examine in what situations computer simulations can exceed clinicians' experience or decisions. In addition, clinicians should learn possible clinical applications and limitations of computational simulations, and engineers should provide this information. Then, clinicians using CFD simulations may provide better treatment to patients in selected situations.

Regarding the second task of the CFD challenge, the specific predictions of the site of rupture were distributed throughout the second aneurysm, with the groups defining 6 different locations. One essential reason might be that only a few hemodynamic studies about rupture points are available. ${ }^{19,20}$ In general, rupturedversus-unruptured aneurysms are compared, but no information regarding the real rupture site can be derived. In addition, hemodynamic studies addressing thin walls and blebs do not necessarily indicate actual rupture sites. Another reason can be seen in the potential level of experience regarding hemodynamic simulations in intracranial aneurysms. Although some groups were highly experienced in other fields of engineering, the survey of the abstracts revealed that unrealistic inflow rates or velocities were applied. For instance, one group defined an inflow velocity of $10 \mathrm{~m} / \mathrm{s}$, whereas another group used a turbulence model that was developed for industrial flows and has not been well-tested on aneurysm-type flows. Another concern is related to the spatial and temporal resolution because some groups used coarse meshes and/or large time-steps in their computations. ${ }^{21}$

Proponents of both the low and the high WSS theory were able to predict the ruptured case. It was found for the group with 
boundary conditions close to the patient-specific that case 2 had lower area-averaged WSS and higher maximum WSS than case 1 (averaged WSS1 $=32.1 \mathrm{dyn} / \mathrm{cm}^{2}$ versus averaged WSS2 $=27.8$ $\mathrm{dyn} / \mathrm{cm}^{2}$; maximumWSS1 $=203.2 \mathrm{dyn} / \mathrm{cm}^{2}$ versus maximumWSS2 $=244.4 \mathrm{dyn} / \mathrm{cm}^{2}$ ). Therefore, the different results (lowversus-high WSS) may not contradict each other. Although at the ruptured site, relatively low WSS and partly elevated OSI were detected, the groups who used these parameters to predict the location did not necessarily succeed in choosing the correct rupture site. Because multiple areas throughout the aneurysm sac fulfilled these conditions, other, even stronger, spots were picked by the corresponding groups (Fig $1 C,-D$ ).

Most interesting, RRT is prolonged on the rupture site and the flow stagnation area on the opposite side. Live images acquired during the intervention revealed the presence of atherosclerosis in this stagnation area, which supports recent findings. ${ }^{17}$ However, no thickening of the aneurysm wall was observed at the rupture site. In that study, ${ }^{17}$ the thick wall had prolonged RRT, but prolonged regions with RRT did not necessarily show a thick wall. Because the results of the second phase ${ }^{15}$ demonstrate that almost all groups are capable of generating nearly identical flow fields under the prescribed boundary conditions, the importance of a realistic numeric setup and model parameters is emphasized once again. Hence, if CFD is integrated in clinical routines some day, guidelines that have evolved from years of experience (eg, spatial and temporal discretization, flow rates, and so forth) should be provided. Such guidelines for hemodynamic simulations in intracranial aneurysms need to address the choice of inflow boundary condition (eg, idealized versus patient-specific ${ }^{22}$ ), flow splitting through multiple outlet sections, wall treatment, blood modeling, and the consideration of pulsatile flow (steady versus unsteady ${ }^{23}$ ), and so forth.

Overall, the following knowledge can be derived from this CFD challenge, which should be considered a snapshot of the current state-of-the-art regarding hemodynamic simulations. Twenty-one of the 26 participants could predict the correct aneurysm and therefore demonstrated that the applied methods are capable of differentiating between the provided cases. Because the particular predictions of the rupture site were relatively diverse, it is not possible to state that CFD can be used to detect the rupture point with high probability currently. To reach this aim, investigating the nature and magnitude of forces that give rise to aneurysm wall remodeling and degradation is necessary. Additionally, CFD should be considered as only 1 aspect besides, for example, patient history or shape analyses within the treatment planning. As a numerical method, it just provides help and not a decision for the attending clinician.

\section{Limitations of the Study}

Although precise predictions of the site of rupture in intracranial aneurysms were performed, this study does not claim that a reliable virtual rupture prediction is possible now. Still, the numeric models underlie too many assumptions, and it is unclear how strongly they affect the real hemodynamics. First, this study involved only 2 aneurysms. Because this study is the first of its kind, participants focused on 2 aneurysms with similar geometric properties and were aware that one had ruptured. This number of cases and prior knowledge are not representative and need to be improved in the future to decide how reliably computational models can predict rupture status. Selected cases from private or open data bases should be considered.

A second limitation is related to the implemented outlet boundary conditions. Some groups applied flow splitting through the different outflows or used Windkessel and resistance models to improve on the assumption of a constant pressure at the outlets. However, the lack of knowledge regarding the real temporal pressure/flow variation at the outlet cross-section certainly leads to inaccuracies of the blood flow simulations.

As a third aspect, rigid vessel walls were assumed for all simulations performed within this study. Although Oubel et $\mathrm{al}^{24}$ found that the actual wall motion does not influence the hemodynamics predicted by CFD and the lack of elastin of aneurysms, which is the essential component to enable flexibility, generally strong differences regarding the wall thickness were observed. ${ }^{25}$ In consequence, the resistance against the imposed flow structures is not equally distributed over the complete aneurysm sac. Therefore, a better understanding of the interaction between the flow and the wall response is required. Vessel wall information such as thickness and strength should be included in future studies if reliable measurements by using, for example, optical coherence tomography or intraoperative microscopy ${ }^{26}$ are possible.

The actual rupture was observed intraoperatively and was thought to be a rerupture of the spontaneous rupture that occurred before the intervention.

Finally, a broad range of skill and experience was detected among the CFD participants, representing a possible state-of-theart of the current status in the field of computational hemodynamics for intracranial aneurysms. The neurosurgeons who completed the questionnaire have years of experience and can be considered experts. This heterogeneity among the biomedical engineers might have an influence on the comparison of both communities.

\section{CONCLUSIONS}

Many groups throughout the world apply numerical methods to investigate cerebral hemodynamics. Challenging these groups by asking them to predict the rupture status of an intracranial aneurysm revealed that approximately $80 \%$ were able to choose the correct aneurysm on the basis of their computations. In particular, low and oscillatory shear was associated with the site of rupture correctly. However, the determination of the rupture site remained challenging if CFD (or, indeed, geometry) was used for the decision exclusively; the predictions were distributed throughout the entire aneurysm sac. Reviewing the participating groups and their submitted abstracts indicates that though great experience concerning engineering problems exists, it might not be sufficient for the investigation of aneurysmal hemodynamics. Therefore, it is of enormous importance that groups have experience in simulating cerebrovascular flows before conclusions are drawn from computational results.

Future challenges in the retrospective prediction of the rupture status in intracranial aneurysms might include more biological aspects. This step will be a necessary one toward the prospective rupture risk assessment. In particular, aneurysm wall 
information and the formation of blood clots or even thrombi should be considered within the numerical modeling. However, because reliable information is not available up to now, the rupture-prediction challenge might be expanded to more cases to test the consistency of the CFD groups.

\section{ACKNOWLEDGMENTS}

The authors express their gratitude to all participants of the CFD Rupture Challenge 2013. The large number of contributions enabled us to reflect the current state-of-the-art in the field of virtual aneurysm research. Additionally, we thank Pedro Lylyk (Interventional Neuroradiology, Clinical Institute ENERI, Buenos Aires, Argentina) and Juan R. Cebral (George Mason University, Fairfax, Virginia) for providing the opportunity to present the results in a plenary session at the IntraCranial Stent Meeting 2013 in Buenos Aires. Furthermore, the authors warmly acknowledge the fruitful discussions with Oliver Beuing (Department of Neuroradiology, University Hospital of Magdeburg, Germany). Special thanks are addressed to Mathias Neugebauer (Department of Simulation and Graphics, University Magdeburg, Magdeburg, Germany) who performed the 3D reconstruction of the patientspecific aneurysms. S.S. thanks Ayako Nadia Aoyagi (Tohoku University Hospital, Japan) for her technical support on this study.

\section{REFERENCES}

1. Bonneville F, Sourour N, Biondi A. Intracranial aneurysms: an overview. Neuroimag Clin N Am 2006;16:371-82, vii

2. Curtis SL, Bradley M, Wilde P, et al. Results of screening for intracranial aneurysms in patients with coarctation of the aorta. AJNR Am J Neuroradiol 2012;33:1182-86

3. Morita A, Kirino T, Hashi K, et al. The natural course of unruptured cerebral aneurysms in a Japanese cohort. $N$ Engl J Med 2012; 366:2474-82

4. Teunissen LL, Rinkel GJ, Algra A, et al. Risk factors for subarachnoid hemorrhage: a systematic review. Stroke 1996;27:544-49

5. Bederson JB, Connolly ES Jr, Batjer HH, et al. Guidelines for the management of aneurysmal subarachnoid hemorrhage: a statement for healthcare professionals from a special writing group of the Stroke Council, American Heart Association. Stroke 2009;40: 994-1025

6. Qian Y, Takao H, Umezu M, et al. Risk analysis of unruptured aneurysms using computational fluid dynamics technology: preliminary results. AJNR Am J Neuroradiol 2011;32:1948-55

7. Takao H, Murayama Y, Otsuka S, et al. Hemodynamic differences between unruptured and ruptured intracranial aneurysms during observation. Stroke 2012;43:1436-39

8. Goubergrits L, Schaller J, Kertzscher U, et al. Statistical wall shear stress maps of ruptured and unruptured middle cerebral artery aneurysms. J R Soc Interface 2012;9:677-88

9. Zhang Y, Mu S, Chen J, et al. Hemodynamic analysis of intracranial aneurysms with daughter blebs. Eur Neurol 2011;66:359-67

10. Xiang J, Natarajan SK, Tremmel M, et al. Hemodynamic-morpho- logic discriminants for intracranial aneurysm rupture. Stroke 2011;42:144-52

11. Cebral JR, Mut F, Raschi M, et al. Aneurysm rupture following treatment with flow-diverting stents: computational hemodynamics analysis of treatment. AJNR Am J Neuroradiol 2011;32:27-33

12. Meng H, Tutino VM, Xiang J, et al. High WSS or low WSS? Complex interactions of hemodynamics with intracranial aneurysm initiation, growth, and rupture: toward a unifying hypothesis. AJNR AmJ Neuroradiol 2014;35:1254-62

13. Xiang J, Tutino VM, Snyder KV, et al. CFD: computational fluid dynamics or confounding factor dissemination? The role of hemodynamics in intracranial aneurysm rupture risk assessment. $A J N R$ Am J Neuroradiol 2014;35:1849-57

14. Steinman DA, Hoi Y, Fahy P, et al. Variability of computational fluid dynamics solutions for pressure and flow in a giant aneurysm: the ASME 2012 Summer Bioengineering Conference CFD Challenge. J Biomech Eng 2013;135:021016

15. Berg P, Roloff C, Beuing O, et al. The Computational Fluid Dynamics Rupture Challenge 2013 - Phase II: variability of hemodynamic simulations in two intracranial aneurysms. J Biomech Eng. In press

16. Neugebauer M, Lawonn K, Beuing O, et al. Automatic generation of anatomic characteristics from cerebral aneurysm surface models. Int J Comput Assist Radiol Surg 2013;8:279-89

17. Sugiyama S, Niizuma K, Nakayama T, et al. Relative residence time prolongation in intracranial aneurysms: a possible association with atherosclerosis. Neurosurgery 2013;73:767-76

18. Kono K, Shintani A, Fujimoto T, et al. Stent-assisted coil embolization and computational fluid dynamics simulations of bilateral vertebral artery dissecting aneurysms presenting with subarachnoid hemorrhage: case report. Neurosurgery 2012;71:E1192-200; discussion E1200-01

19. Kono K, Fujimoto T, Shintani A, et al. Hemodynamic characteristics at the rupture site of cerebral aneurysms: a case study. Neurosurgery 2012;71:E1202-08; discussion 1209

20. Omodaka S, Sugiyama S, Inoue T, et al. Local hemodynamics at the rupture point of cerebral aneurysms determined by computational fluid dynamics analysis. Cerebrovasc Dis 2012;34:121-29

21. Valen-Sendstad K, Steinman DA. Mind the gap: impact of computational fluid dynamics solution strategy on prediction of intracranial aneurysm hemodynamics and rupture status indicators. $A J N R$ Am J Neuroradiol 2014;35:536-43

22. Jansen IG, Schneiders JJ, Potters WV, et al. Generalized versus patient-specific inflow boundary conditions in computational fluid dynamics simulations of cerebral aneurysmal hemodynamics. AJNR Am J Neuroradiol 2014;35:1543-48

23. Babiker MH, Gonzalez LF, Albuquerque F, et al. An in vitro study of pulsatile fluid dynamics in intracranial aneurysm models treated with embolic coils and flow diverters. IEEE Trans Biomed Eng 2013;60:1150-59

24. Oubel E, DeCraene M, Putman CM, et al. Analysis of intracranial aneurysm wall motion and its effects on hemodynamic patterns. Proc SPIE 2007;6511:65112A

25. Frösen J, Tulamo R, Paetau A, et al. Saccular intracranial aneurysm: pathology and mechanisms. Acta Neuropathol 2012;123:773-86

26. Kadasi LM, Dent WC, Malek AM. Cerebral aneurysm wall thickness analysis using intraoperative microscopy: effect of size and gender on thin translucent regions. J Neurointerv Surg 2013;5:201-06 